\title{
e-Phaïstos
}

e-Phaïstos

Revue d'histoire des techniques / Journal of the history

of technology

VII-2 | 2019

La sauvegarde du patrimoine scientifique

\section{La clarinette logique. Inventaire et sauvegarde d'un instrument scientifique en développement.}

Research Instruments Heritage (second half of the 20th and beginning of the 21st century) : Inventory and Conservation of Scientific Instruments in Development

\section{Corine Lévy-Soussan}

\section{(2) OpenEdition}

\section{Journals}

Édition électronique

URL : http://journals.openedition.org/ephaistos/5429

DOI : 10.4000/ephaistos.5429

ISSN : 2552-0741

Éditeur

IHMC - Institut d'histoire moderne et contemporaine (UMR 8066)

Référence électronique

Corine Lévy-Soussan, « La clarinette logique. Inventaire et sauvegarde d'un instrument scientifique en développement. », e-Phaïstos [En ligne], VII-2 | 2019, mis en ligne le 15 octobre 2019, consulté le 10 décembre 2020. URL : http://journals.openedition.org/ephaistos/5429 ; DOI : https://doi.org/10.4000/ ephaistos.5429

Ce document a été généré automatiquement le 10 décembre 2020.

Tous droits réservés 


\section{La clarinette logique. Inventaire et sauvegarde d'un instrument scientifique en développement.}

Research Instruments Heritage (second half of the 20th and beginning of the 21st century) : Inventory and Conservation of Scientific Instruments in Development

\section{Corine LÉVY-SOUSSAN}

\section{Introduction}

1 L'objet de cet article ${ }^{1}$ est de proposer une méthode et un modèle de fiches d'inventaire adaptés aux instruments scientifiques en développement ${ }^{2}$. Nous avons choisi pour illustrer cette méthode, un instrument récent et en cours d'étude, 'la clarinette logique' 3, qui doit permettre au musicien d'obtenir aisément la justesse du son. La méthode employée pour constituer la fiche d'inventaire prend en compte l'évolution des différents composants de l'instrument ainsi que la synthèse de la collecte de témoignages oraux, indispensables pour la composition du dossier d'inventaire et la compréhension des instruments contemporains. La sauvegarde et la conservation de l'objet matériel ne sont pas en effet dissociables des pratiques sociales et des témoignages qui font partie intégrante du contexte dans lequel l'objet matériel est conçu et utilisét. La question est de savoir comment l'inventaire prendra en compte toutes ces données. Les instruments en développement relèvent d'une approche spécifique qui a pu être expérimentée mais on reste au stade du prototype. Ce modèle de fiche s'adresse aux experts, mais également au grand public et pourrait également être un outil pour l'enseignement. Par ailleurs, ce travail d'inventaire nous a permis d'identifier une 'lignée technique ${ }^{5}{ }^{\prime}$ 'instruments scientifiques, celle des résonateurs acoustiques dont l'origine remonte à la plus haute antiquité, l'Égypte ancienne, ainsi que le régime ${ }^{6}$ et les ruptures technologiques dans le domaine de l'acoustique. Nous nous interrogerons enfin sur la dématérialisation du patrimoine et sur la pérennité des 
données puisqu'on ne peut échapper à la question de la durée du stockage et de la conservation des données.

\section{Problématiques de l'inventaire et de la conservation du patrimoine scientifique et technique}

\section{La mission nationale du patrimoine scientifique et technique contemporain (PATSTEC) d'Aix-Marseille Université}

La préservation, la conservation et la valorisation du patrimoine scientifique et technique contemporain est un sujet qui fait débat depuis plus d'une quinzaine d'années. Les instruments scientifiques utilisés dans les universités ou dans les laboratoires de recherche ne sont plus conservés. Dès les années 1950, ils sont victimes de la rapidité d'évolution des technologies et devenus obsolètes, sont souvent jetés car la science s'intéresse avant tout aux concepts et aux résultats. Dans ce contexte AixMarseille Université (AMU) en région Provence-Alpes-Côte d'Azur, s'est associée à la mission nationale du PATSTEC (Patrimoine scientifique et technique contemporain) pour le recensement du matériel des laboratoires de recherche. Le périmètre de notre recherche se situe dans le cadre du chantier d'inventaire en 2017, du Laboratoire de Mécanique et d'Acoustique (LMA) à Marseille dont l'histoire remonte aux années 1920 à Toulon, sous le nom du «Centre d'Études de Toulon » (LCET).

3 L'inventaire des instruments du LMA établi sur plus d'une centaine d'instruments est accessible sur la base de données du PATSTEC. La collection informatisée sur la base de données du PATSTEC permet de garder en mémoire les objets susceptibles de disparaître. Il faut cependant garder à l'esprit que la complexité des instruments scientifiques pose toute une série de problèmes relatifs à leur conservation, à leur étude et à leur inscription dans une base de données numérique : « La fiche descriptive d'un instrument contemporain, risque « d'exploser » dans les mains de son compilateur sans pour autant pouvoir être complète et exhaustive ${ }^{7}$ ». Des questions sensibles se posent aussi sur la pérennité des données et concernant la gestion patrimoniale, comme l'enregistrement, le stockage d'une grande quantité de données et sur la durée de vie des supports numériques, toutes choses qui font l'objet de nombreux débats.

\section{La méthode d'inventaire de la mission PATSTEC}

La personne chargée d'inventaire utilise toujours une méthode qui consiste à faire des enquêtes auprès des chercheurs, sous forme de questionnaires appliquées aux instruments qui marquent la fin d'une recherche et dont le développement est terminé. Cette méthode comprend le patrimoine matériel, qui va de l'instrument au produit industriel, les manuscrits, les documents associés et le patrimoine immatériel: la mémoire vivante recueillie sous forme d'interviews, les savoir-faire des hommes et des femmes de la recherche, qui ont utilisé ces objets. La procédure d'inventaire dite " classique » s'adresse à une catégorie d'instruments identifiée comme étant «stable». Nous pouvons dire que l'instrument est stabilisé et qu'il peut être décrit avec toutes ses caractéristiques définies et définitives. La fiche d'inventaire du PATSTEC présente un grand nombre de champs d'informations spécifiques aux objets scientifiques et techniques de la recherche contemporaine : intérêt scientifique de l'objet, son histoire, 
la description physique, son mode de fonctionnement, le nom du constructeur, l'année de fabrication, l'état de son fonctionnement, les mesures, les photographies et le contexte de l'objet. Elle aboutit à une fiche de recueil de données stables.

Ce travail est réalisé sur des archives et en général avec un correspondant scientifique qui est souvent un chercheur ou un ingénieur en fin d'activité professionnelle. Le matériel n'étant plus utilisé pour la recherche, l'état final est conservé, étiqueté et inventorié. Un grand nombre d'instruments des années 1950 à 2000 du LMA est ainsi identifié comme étant stable parce qu'il arrive au bout d'un programme de recherche. Ces instruments devenus obsolètes qui entrent dans une collection ont plusieurs origines. Ils peuvent représenter une expérience qui a marqué une étape dans la recherche ou bien avoir été utilisés à une certaine époque comme simple instrument de mesure. L'essence même d'un instrument est de mettre en évidence un phénomène physique prouvant ainsi une certaine "réalité ». Comprendre le fonctionnement de l'ensemble de la conception à la réalisation et l'associer à la façon de faire la science, permet de mieux percevoir la particularité de l'instrument en question.

\section{Le cas des instruments en développement et la problématique de leur inventaire}

6 Pour l'inventaire, un problème se pose lorsqu'il s'agit d'un dispositif matériel «en développement", partie prenante d'une recherche en cours. Les fiches d'inventaire " classiques » présentent en effet des objets qui n'évoluent plus et ne sont pas adaptées au cas d'objets en développement. Les instruments des années 2000 à aujourd'hui (scanner 3D, becs instrumentés, bouches artificielles pour clarinette, prototype de clarinette logique, synthèse sonore, etc..), sont toujours utilisés dans le laboratoire par les enseignants-chercheurs, ingénieurs ou techniciens et sont en constante évolution. Ces instruments du patrimoine « en train de se construire » sont modifiés, en fonction des questions que se posent les chercheurs et aussi en fonction de l'évolution des technologies, générant sans cesse différentes versions de l'instrument.

7 Ce sont ces différentes étapes de la modification, même minimes de ces instruments, qui témoignent de la dynamique de la recherche sur laquelle ils reposent et à laquelle ils contribuent. Nous avons trouvé des exemples semblables à celui de notre étude de cas au Laboratoire d'Astrophysique de Marseille (LAM) durant l'inventaire effectué en 2018. Ceci a conforté le souhait d'avancer sur une méthode d'inventaire adaptée aux instruments en développement. L'enjeu est considérable, puisqu'il s'agit en quelque sorte de sauvegarder la mémoire de la recherche "en train de se faire ». Comment peut-on l'envisager? L'historique, l'organisation du laboratoire et le témoignage des chercheurs vont apporter des éléments, au-delà de ce que contiennent les cahiers de laboratoire. Le patrimoine du laboratoire ne se limite pas, en effet, à l'aspect matériel de la recherche, il est présent aussi dans les traces immatérielles (histoire du laboratoire, activités de recherche, témoignages, gestes, vocabulaire, habitudes, 'manip's'...) :

«Une approche prometteuse consiste à travailler avec les chercheurs eux-mêmes, en les observant au sein de leur laboratoire, en les amenant à parler des objets qu'ils manipulent, des gestes qu'ils font sans plus le savoir, mais qui sont constitutifs de leurs « manip's ${ }^{8}{ }^{8}$. 
8 L'instrument scientifique est indissociable d'une réalité immatérielle, patrimoine qui risque de se perdre s'il n'est pas traité en même temps que l'instrument lui-même. Ceci passe par la compréhension du fonctionnement de l'ensemble, de la conception à la réalisation et de son association à la façon de faire la science. A partir d'entretiens avec les chercheurs nous avons constitué un corpus de données et réalisé des fiches synthétiques d'un instrument particulier: la clarinette (clarinette logique, synthèse sonore, bec instrumenté et bouche artificielle).

9 Se pose alors, dans ce contexte, la question de savoir quelles informations garder pour ensuite les contextualiser et transmettre ces connaissances de culture scientifique et technique aux professionnels, aux corps enseignants ou à un large public. Ceci renvoie à des interrogations sur ce qu'on cherche à sauvegarder, des objets techniques et/ou des concepts, la culture d'un laboratoire, des témoignages matériels et immatériels de la recherche, des gestes techniques, tout cela à la fois? Mais alors, est-il possible de proposer une méthode d'inventaire et un protocole adapté aux instruments de la recherche en développement qui prenne en compte ces éléments, avec quelles limites et quels protocoles avec les chercheurs? Se posent en outre des questions spécifiques pour l'inventaire des objets techniques incorporant des logiciels et des capteurs. Autrement dit il est question de savoir comment patrimonialiser l'objet numérique contemporain.

\section{Étude du cas : la clarinette logique pour la synthèse sonore}

10 Nous avons choisi d'appliquer cette méthode à un instrument en développement, à savoir la clarinette logique et la synthèse sonore actuellement utilisées (et modifiées), ceci afin d'étudier en détail le problème de l'inventaire et de la sauvegarde du patrimoine instrumental contemporain. Précisons ici que la clarinette logique est constituée d'un corps cylindrique percé de trous : le résonateur. Il est excité par le jet d'air oscillant modulé par les vibrations de l'anche simple vibrante fixée au bec. 
Figure 1. Prototype de la clarinette logique (CAGIMA, projet de recherche fondamentale) dont les différentes parties de l'instrument sont construites par modélisation, (Laboratoire de mécanique et d'acoustique), 2017, fiche inventaire PATSTEC

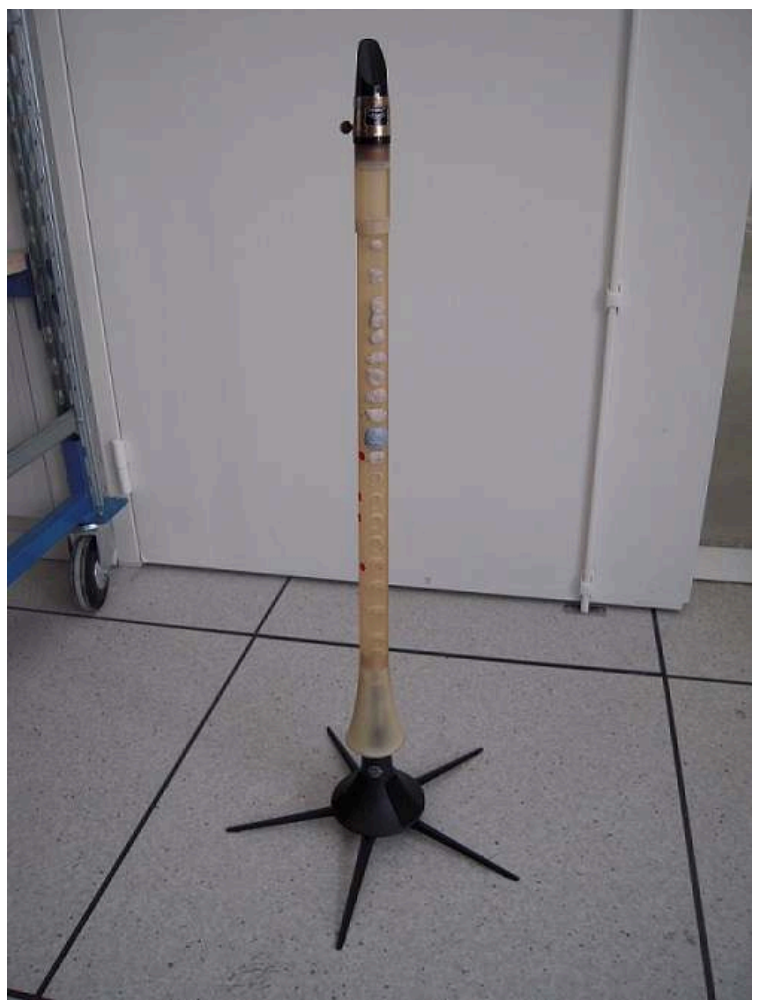

Le prototype de clarinette logique est en perpétuelle évolution, objet unique au monde, il se présente comme un bout de plastique mais cela a donné lieu à des années de recherche. Il est réalisé par imprimante 3D au LMA et fonctionne comme une clarinette acoustique, c'est à dire jouée par un musicien. II est fabriqué après la réalisation de tests numériques et conçu par des calculs en modélisant les composants de l'instrument. Ces données sont prises en compte par le fabricant d'instruments pour la réalisation matérielle de la clarinette. Le corps de la clarinette se comporte comme un résonateur dont les caractéristiques acoustiques sont très fortement dépendantes de la perce de l'instrument (cylindrique, faiblement conique ou fortement conique comme le saxophone) et aux trous latéraux définissant le doigté de l'instrument. La flûte ou la clarinette ont énormément évolué depuis la période baroque. Les plus récentes connaissances en acoustique, les possibilités de calcul liées à la puissance exponentielle de l'informatique permettent aujourd'hui d'optimiser ces paramètres acoustiques (justesse, facilité d'émission, puissance, la quête étant la détermination d'un timbre voulu) sous la forme d'instruments 'logiques' de façon plus systématique que l'empirisme de la facture instrumentale.

(c) Corine Lévy Soussan

\section{Le programme de recherche " clarinette logique » du LMA et ses deux projets}

11 Les activités de recherche de l'équipe Sons se rattachent aux domaines des vibrations, vibroacoustique, aéroacoustique, audition, psychoacoustique, musique, audio et informatique en temps réel. Dans le domaine de la musique une partie de l'équipe Sons du LMA travaille sur deux projets complémentaires :

- le premier est la 'conception acoustique globale d'instruments de musique à anches justes et homogènes' « Le prototype de clarinette logique sert alors à démontrer la validité d'un procédé de conception d'un instrument de musique acoustique, entièrement par prototypage virtuel ${ }^{9} \%$. Il est réalisé par imprimante 3D au LMA. La clarinette fonctionne alors comme une clarinette acoustique, c'est à dire jouée par un 
musicien.

- le second projet concerne la Synthèse sonore qui a deux objectifs ${ }^{10}$ : d'une part le développement des algorithmes qui servent à tester les prototypes de la clarinette logique et d'autre part la production des sons artificiels.

Philippe Guillemain ${ }^{11}$ qui est à l'origine de cet instrument « parfait », nous explique que la conception de cet instrument et de ses éléments (bec et bouche artificielle) permet au musicien de jouer en obtenant la justesse des sons (moins de trous et de clétages que dans le passé). Ce projet a été développé en collaboration avec l'entreprise Buffet Crampon, créée en 1825. Le groupe «Son » du LMA travaille avec cette entreprise sur la conception globale d'instruments à anches à trous latéraux "plus justes et plus homogènes» que nous appellerons instruments "logiques». Ce travail a été appliqué à un instrument de type clarinette dont le corps est constitué d'un cylindre muni de trous latéraux. (Projet CAGIMA).

\section{Le bec de la clarinette}

Le bec contient le générateur de fréquence constitué de l'anche en vibration et le jet d'air oscillant ${ }^{12}$.

Figure 2. Le bec instrumenté pour clarinette en 3D, (Laboratoire de mécanique et d'acoustique), 2017, fiche inventaire PATSTEC.

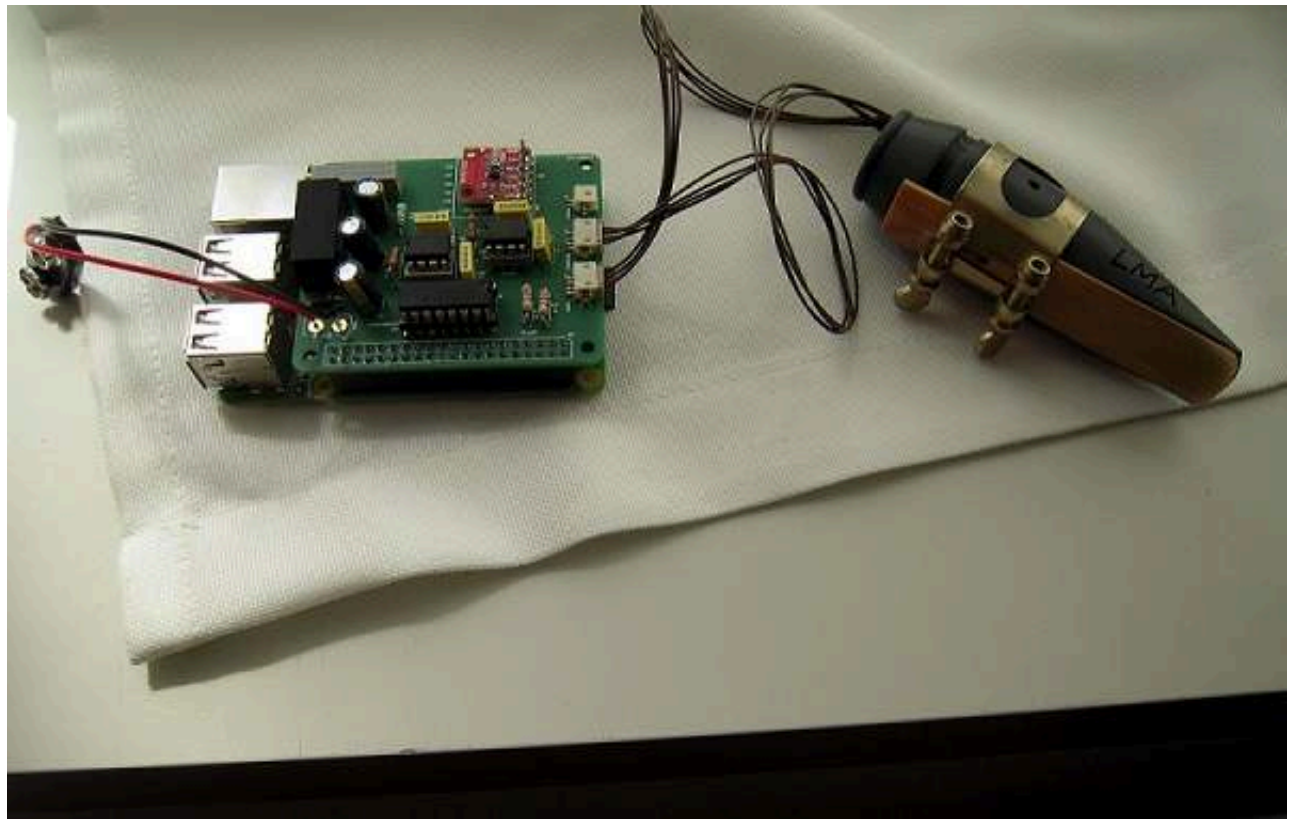

Le concept de becs instrumentés date d'avant 2008. Plusieurs pays ont fabriqué des becs dans les laboratoires (Canada, Australie). Les différentes mesures, pression dans la bouche du musicien, force d'appui de la lèvre sur l'anche, déplacement de l'anche sont réalisées à l'aide du bec instrumenté, c'est à dire équipé de capteurs. La série des becs instrumentés de la clarinette est à considérer comme une " série technologique » qui évolue sous des contraintes extérieures : évolution des capteurs, évolution des logiciels, possibilité de réaliser des becs avec une imprimante 3D.

(c) Corine Lévy Soussan

14 Le bec instrumenté sert à mesurer les paramètres de jeu d'un musicien jouant d'un instrument à anche simple (clarinette, saxophone). Il se présente sous la forme d'un bec réalisé en impression 3D au LMA accompagné d'une carte électronique (Raspberry PI 3) et d'une carte de conditionnement des signaux conçue au LMA. Les paramètres sont 
l'ouverture de l'anche et la pression dans le bec. Ces 2 paramètres sont envoyés par wifi selon le protocole OSC (Open Sound Control) au programme de modélisation physique qui s'exécute en temps-réel sur la tablette ou le smartphone. Le son est généré en fonction de l'évolution temporelle des deux paramètres.

Figure 3. Gros plan du bec instrumenté, (Laboratoire de mécanique et d'Acoustique), 2017, fiche inventaire PATSTEC.

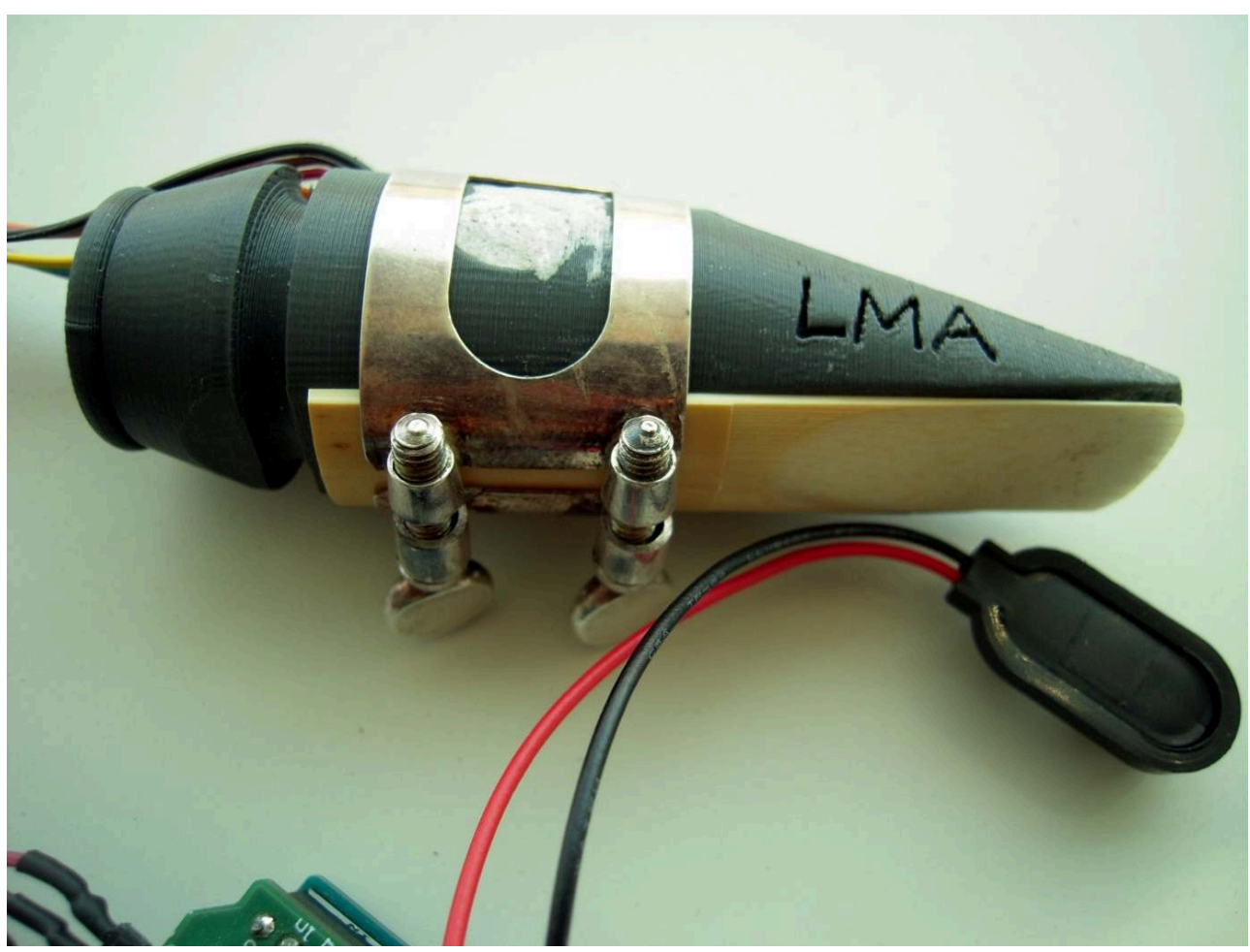

(c) Corine Lévy Soussan

\section{La bouche artificielle}

La bouche artificielle est une machine à souffler et à reproduire les fonctions essentielles d'un musicien, ce qui permet d'avoir des instruments qui jouent sans musicien ${ }^{13}$. Pour les besoins de la physique appliquée aux instruments de musique à vent, les scientifiques ont développé des bouches artificielles afin de maitriser les paramètres principaux des modèles d'acoustiques, dispositif supposé répétable permettant d'étudier, dans un premier temps, le seuil d'émission du son. Ces bouches artificielles permettent aussi d'étudier les régimes de bifurcation produisant par exemple un changement d'octave en faisant intervenir la notion de vitesse, pression et effets dynamiques de l'air injecté et ceci pour chaque note. 
Figure 4. La bouche artificielle, (Laboratoire de mécanique et d'acoustique), 2017, fiche inventaire PATSTEC

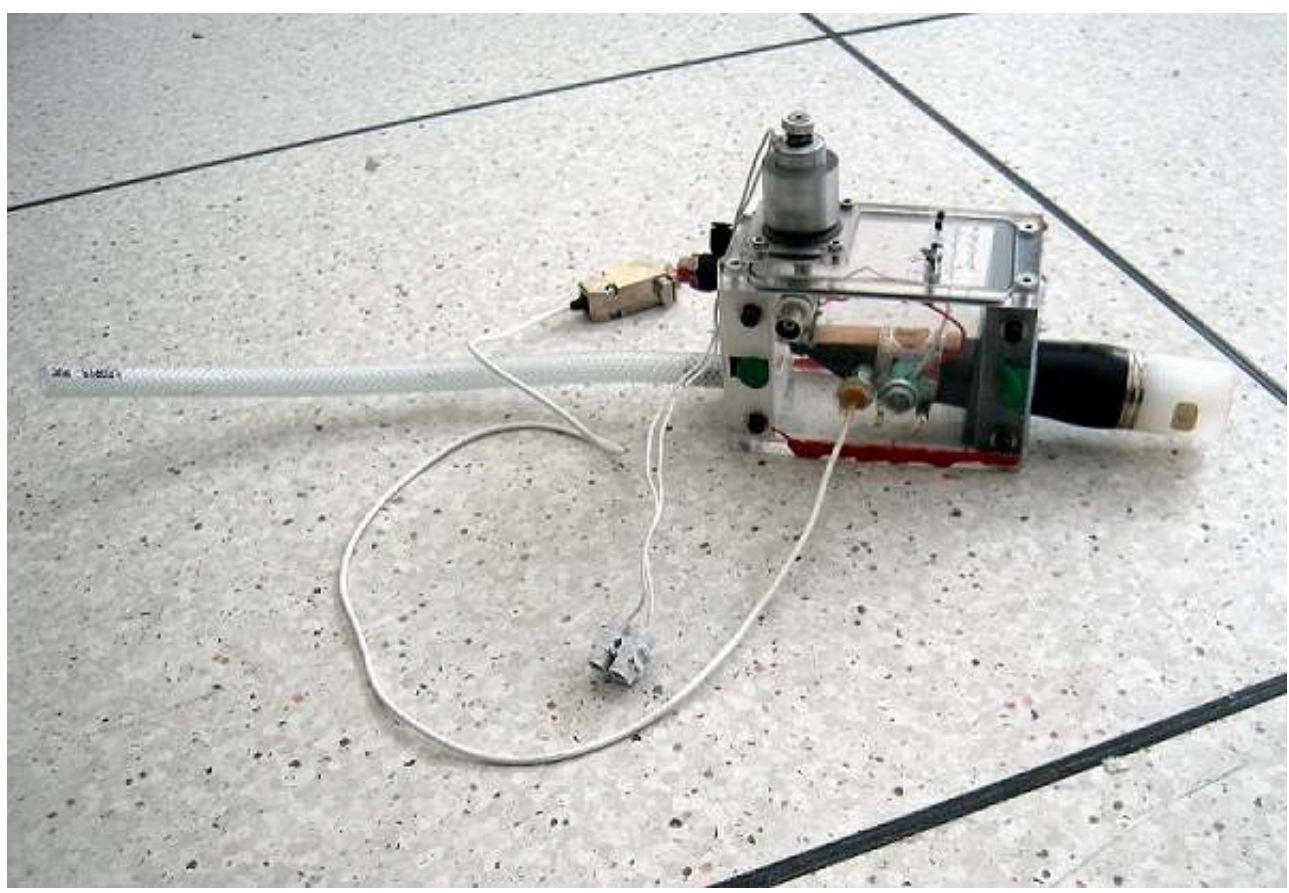

Les bouches artificielles sont équipées de capteurs qui mesurent en temps réel la manière dont l'instrument réagit indépendamment du musicien, par exemple comment la hauteur de la note évolue en fonction de la pression d'alimentation. Les signaux mesurés sont analysés pour comprendre comment les paramètres contrôlés interviennent dans la production du son. Un compresseur envoie de l'air vers les poumons, ici remplacés par un volume équivalent. Des tuyaux de diverses sections et longueurs simulent la trachée, la glotte, le conduit vocal et la bouche. Les lèvres sont remplacées par des tubes en latex dont la pression est contrôlée par une colonne d'eau. Les premières bouches artificielles datent de 1940 aux États-Unis (USA) et dans d'autres pays en 1960. Les premiers modèles des bouches artificielles pour cuivres existent depuis 1997 à l'IRCAM et la seconde génération en 2008. La dernière version évolue depuis une quinzaine d'années. Pour les besoins de la physique appliquée aux instruments de musique à vent, les scientifiques ont développé des bouches artificielles afin de maitriser les paramètres principaux des modèles d'acoustiques, dispositif supposé répétable permettant d'étudier, dans un premier temps, le seuil d'émission du son. Ces bouches artificielles permettent aussi d'étudier les régimes de bifurcation produisant par exemple un changement d'octave en faisant intervenir la notion de vitesse, pression et effets dynamiques de l'air injecté et ceci pour chaque note.

(c) Corine Lévy Soussan

\section{Le premier projet : la clarinette logique}

Le corps de la clarinette ${ }^{14}$ sélectionne les fréquences musicales, c'est le résonateur percé de trous. Il se comporte donc comme un résonateur dont les caractéristiques acoustiques sont très fortement dépendantes de la perce ${ }^{15}$ de l'instrument (cylindrique, faiblement conique ou fortement conique comme le saxophone). La caractéristique d'un instrument logique « est de ne pas comporter de trous fermés en aval du premier trou ouvert comme c'est le cas pour la clarinette actuelle. Ce critère signifie que l'effort d'adaptation du geste du musicien doit être minimal, sans émettre successivement deux notes avec la même justesse et la même nuance musicale, d'où le lien avec le caractère logique de l'instrument $\aleph^{16}$. Ces éléments de perçage ${ }^{17}$ (forme interne de perce) et d'ouvertures (doigtés) ont fait l'objet de nombreuses évolutions dans le temps, afin d'améliorer la facilité du jeu, la justesse, la puissance du son produit. La flûte ou la clarinette ont énormément évolué dans ce sens depuis la période baroque. Les plus 
récentes connaissances en acoustique, les possibilités de calcul liées à la puissance exponentielle de l'informatique permettent aujourd'hui d'optimiser ces paramètres acoustiques (justesse, facilité d'émission, puissance, la quête étant la détermination d'un timbre voulu) sous la forme d'instruments 'logiques' de façon plus systématique que l'empirisme de la facture instrumentale. Mais avant de construire un prototype, les chercheurs mesurent l'impédance acoustique c'est-à-dire la manière dont réagit en pression le tuyau sonore excité par un débit d'air à une fréquence donnée : le corps de la clarinette se comporte comme un résonateur dont on peut calculer la réponse à partir de la mesure de son impédance d'entrée. Cette impédance interviendra dans le cadre de la synthèse sonor $\mathrm{e}^{18}$. Notons ici une nouvelle lignée technique : les résonateurs acoustiques. A partir de ces résultats plusieurs prototypes ont été réalisés et testés par les musiciens essayeurs du facteur Buffet Crampon.

Les trois équations du modèle numérique du $\mathrm{bec}^{19}$ :

\begin{abstract}
«Le prototype de la clarinette logique est conçu à partir des calculs modélisant les composants de l'instrument. Ces données sont prises en compte par le fabricant d'instruments pour la réalisation matérielle de la clarinette. Concernant le bec, cette modélisation conduit à écrire trois équations qui vont par la suite servir de socle à la reproduction par ordinateur du son de la clarinette. La clarinette est à son tour un objet d'étude visant à en concevoir une autre ».
\end{abstract}

Les trois équation $\mathrm{s}^{20} \mathrm{du}$ modèle global du bec qui mènent à un procédé numérique de simulation d'un instrument se présentent de la manière suivante :

- la première équation concerne la géométrie de l'excitateur et décrit le débit d'air à travers ses constituants (relation pression-débit);

- la deuxième équation décrit les vibrations de l'anche simple seule activée par la différence de pression entre la bouche et l'entrée du résonateur. C'est cette équation qui relie le débit d'air aux fréquences qui seront ensuite émises par le résonateur (corps de l'instrument);

- la troisième équation représente la caractéristique non linéaire grâce à laquelle peuvent s'établir les auto-oscillations produisant le son. Elle exprime le débit acoustique qui agit comme la membrane d'un haut-parleur et va exciter le résonateur en fonction de son impédance d'entrée que l'on peut modifier à l'aide du clétage pour produire différentes notes. L'impédance d'entrée du résonateur peut être mesurée par un capteur d'impédance ou calculée.

Ces trois équations servent à tester la clarinette logique par la production de sons. C'est un prototype unique destiné à valider et invalider des hypothèses de conception. Elle est fabriquée après la réalisation de tests numériques. Si les tests numériques ne sont pas concluants, l'équipe conçoit un nouveau prototype numérique en modifiant les critères. Les algorithmes de la synthèse sonore et la création de sons artificiels par ordinateur, servent à tester le prototype de la clarinette logique.

\title{
Le second projet : la synthèse sonore ${ }^{21}$
}

20 La 'synthèse sonore' est composée d'un logiciel de simulation écrit à partir des trois équations du bec et des équations de l'impédance d'entrée de la clarinette, intégré à un 
ordinateur et d'un capteur gestuel muni d'une boite de contrôle, d'un amplificateur et d'un haut-parleur.

Cet instrument (logiciel, ordinateur et contrôleur) a pour fonctions de tester les prototypes de la clarinette logique et de produire des sons artificiels,

Figure 5. La synthèse sonore : ordinateur portable accueillant l'environnement logiciel de synthèse constitué d'un capteur gestuel de commerce, Laboratoire de mécanique et d'acoustique, 2017.

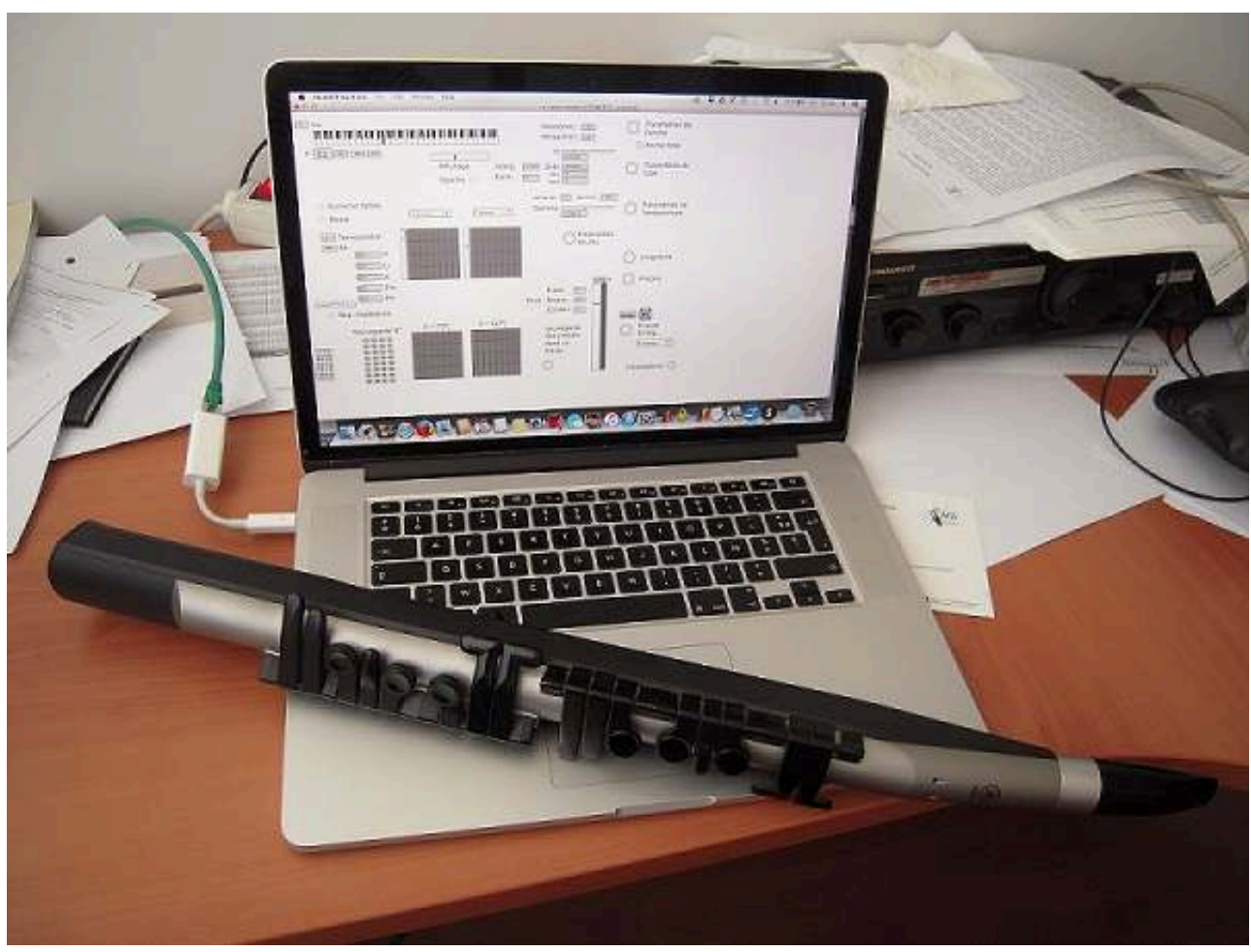

La Synthèse sonore a deux objectifs : d'une part le développement des algorithmes qui servent à tester les prototypes de la clarinette logique et d'autre par la production des sons artificiels. La production de sons grâce à l'ordinateur ou « synthèse sonore » occupe depuis les années 50 une place importante parmi les outils dont dispose le musicien. La synthèse sonore repose sur des modèles physiques de fonctionnement et sur son application à la facture instrumentale. II s'associe aussi aux recherches portant sur le lien « geste-son » dans la production sonore instrumentale. Ce n'est qu'à partir des années 20 que la technologie a permis de créer des sons artificiels à l'aide des composants électroniques simples (principalement des oscillateurs). C'est alors que l'on a commencé à parler de synthèse sonore. Elle peut s'appuyer sur des modèles de signaux (le résultat sonore) ou sur des modèles physiques de fonctionnement (la cause de la production du son).

(c) Corine Lévy Soussan

22 Le logiciel Max/MSP22 a permis de construire l'interface utilisateur de contrôle de l'algorithme de synthèse temps-réel codé en langage $C$. Le contrôle s'effectue à deux niveaux. Le premier concerne les paramètres décrivant le modèle géométrique simplifié de résonateur et les caractéristiques de l'excitateur, c'est-à-dire ceux qui vont différencier par exemple une clarinette d'une trompette. Le deuxième niveau de contrôle gère le lien avec les données de jeu musical issues du capteur gestuel qui pilotent dynamiquement l'algorithme de synthèse.

La partie informatique fait donc l'objet de deux projets, l'un pour créer l'instrument acoustique (clarinette logique) et l'autre pour créer des sons artificiels. Grâce à la résolution d'un système de calcul (trois équations à trois inconnues), on stimule par ordinateur, le fonctionnement de la clarinette logique. La démarche des concepteurs de 
ces instruments consiste en la capacité de penser un ensemble technologique dans un avenir proche comme prévoir les conditions de sa production, de son usage et de sa réussite commerciale.

Les projets réalisés dans les laboratoires sont ensuite développés et commercialisés par une entreprise. Mais les musiciens vont-ils s'emparer de la clarinette logique sachant que les instrumentistes sont attachés aux matériaux naturels de l'instrument (bois) et à la méthodologie employée par les facteurs d'instruments qui est essentiellement empirique?

\section{Les apports des méthodes de l'histoire des techniques : la notion de lignée technique et le régime technologique}

\section{Définitions}

Suite à ce travail se sont posées les questions de l'inventaire des instruments qui évoluent et qui disparaissent et l'objet devient accessoire au profit des processus de production de connaissances scientifiques. Est-ce que ces instruments sont une source de connaissance pour l'histoire des techniques?

«L'histoire des objets techniques est l'une des composantes de l'histoire des techniques ${ }^{23}$. L'évolution de la lignée des objets techniques fait partie du programme d'enseignement dans les lycées. L'aspect évolutif des objets techniques qui intéresse de nombreuses disciplines scientifiques, ouvre des perspectives pour la gestion des connaissances. Notre cas d'instrument pourrait se distinguer selon le point de vue qui oppose la cohérence horizontale et verticale ${ }^{24}$. La première répond aux enjeux de classification des objets, la seconde aux grandes mutations des techniques. Dans cette perspective, les ruptures technologiques sont identifiées et analysées.

Anne-Françoise Garçon définit une lignée technique par l'usage, le fonctionnement et l'ensemble des activités productrices qui sont liées à l'objet technique qui lui permettent d'exister. Les travaux développés ${ }^{25}$ sur la genèse de l'objet technique concernent les origines de l'objet. Cette technicité relève de l'usage et donc du fonctionnement autonome associé à son milieu technique. Les éléments communiquent les uns avec les autres. La lignée se définit donc par son fonctionnement. C'est ce point de vue que nous adopterons : les objets sont classés d'après leur fonction et leur but. L'inventaire des instruments scientifiques du LMA nous a permis de construire une lignée technique en partant de l'histoire des résonateurs acoustiques. La plupart des instruments anciens et actuels utilisent des résonateurs acoustiques (instruments à vent) et mécaniques (instruments à cordes, percussions). Dans la lignée technique des résonateurs, le corps de la clarinette est un tuyau résonant, perforé de trous que l'on ouvre, que l'on ferme à l'aide du clétage afin d'obtenir les différentes notes musicales. Le corps de la clarinette est excité par l'anche en vibration et le jet d'air oscillant qui se trouve dans le bec et c'est le jeu du musicien qui permet de produire différentes notes de musique. La lignée technique des instruments scientifiques du LMA commence par les résonateurs de Helmholtz. La plupart des instruments anciens et actuels utilisent des résonateurs acoustiques (instruments à vent) et mécaniques (instruments à cordes, percussions). 


\section{Application au cas de la clarinette logique}

Le socle commun au développement de la clarinette logique et à celui de la synthèse sonore est constitué ${ }^{26}$ :

- de la mécanique des fluides (pour le bec) et de l'acoustique (pour le résonateur),

- des outils mathématiques qui ont permis le développement de modèles de plus en plus précis autorisés par la puissance du calcul numérique,

- des expérimentations numériques des modèles physiques en temps réels (le calcul à grande vitesse et le contrôle des capteurs).

Le chercheur entend par " prototypage virtuel » la simulation d'un résultat sonore d'un modèle numérique créé suivant les critères des instruments à vent. Cette démarche présente l'intérêt d'avoir le résultat sonore, concret d'un instrument imaginé et modélisé d'une façon informatique. Ce procédé permet d'obtenir un résultat avant la fabrication de l'instrument, et d'éliminer les impasses sur des modèles non réalistes en qualité d'ergonomie ou en qualité du son médiocre (y compris la justesse). Le prototypage permet aussi l'étude et la modélisation d'instruments déjà existants et réalisés de façon empirique (certains vieux instruments). En effet il devient possible grâce à cette technique de comprendre le comportement physique de l'instrument, d'y trouver parfois des erreurs de conception, c'est ainsi qu'est née l'idée de corriger ces défauts et de produire une clarinette corrigée.

Héritière du même corpus théorique que sa consœur la clarinette logique, la clarinette de synthèse intègre un modèle sous forme numérique. Nous sommes ici en présence d'une bifurcation possible de la lignée technique de la clarinette classique ou logique vers la lignée des synthétiseurs avec la possibilité d'obtenir le son de la clarinette ${ }^{27}$. Le point clé est que le système analogique (clarinette classique et logique) dit en temps réel, n'a pas de temps de latence ${ }^{28}$. Par contre, la lignée des synthétiseurs connaît depuis de nombreuses années ce problème de latence. C'est ainsi que nous pouvons parler de bifurcation du fait que les synthétiseurs fonctionnent presque en temps-réel mais pas en temps réel. Il est à noter que cette latence mobilise toutes les énergies créatrices des développeurs dans le domaine de la synthèse numérique pour qu'elle tende vers zéro. Ce décalage doit rester à des seuils d'une dizaine de millisecondes pour que l'instrument reste jouable. Malheureusement plus on augmente le volume du calcul numérique plus le délai de restitution du son prend du temps. 
Figure 6 : Carte heuristique de la lignée technique de la clarinette en l'état de l'art, réalisation, capture d'écran réalisé le 10 juillet 2018.

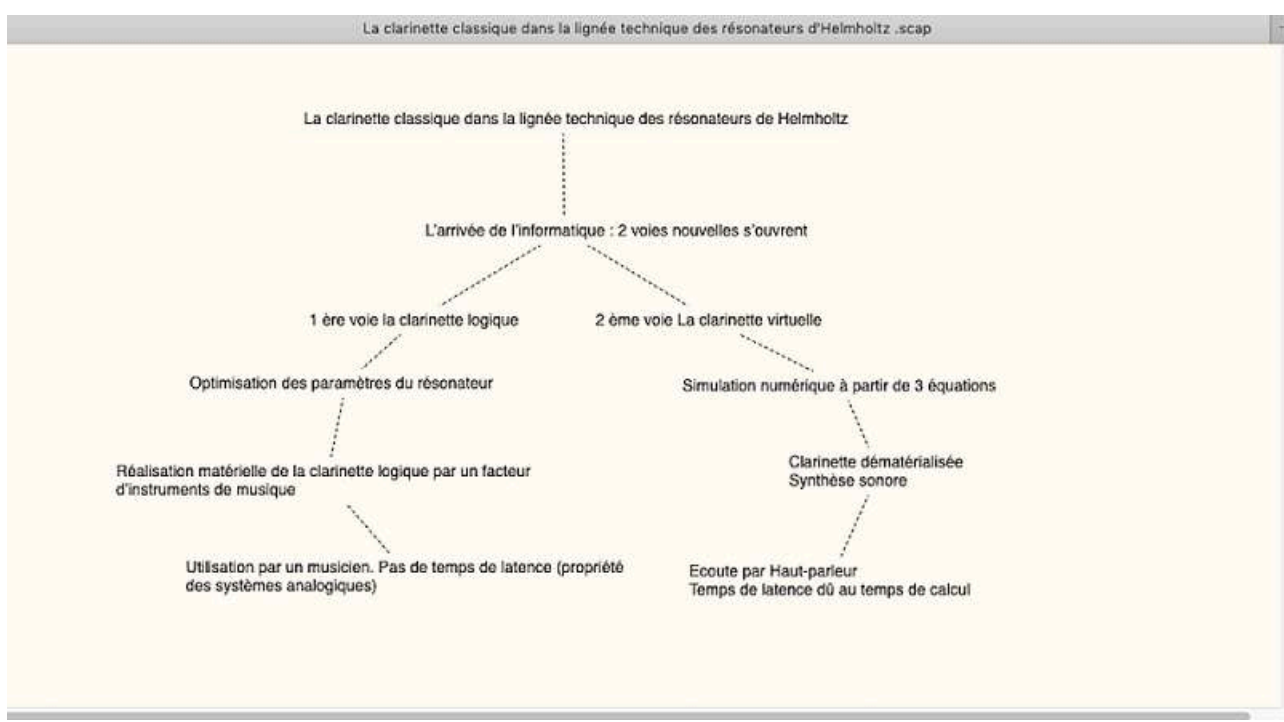

La carte heuristique montre que nous sommes en présence d'une bifurcation de la lignée technique de la clarinette traditionnelle qui repose sur la théorie des résonateurs, partie essentielle des analyseurs de fréquence. Les systèmes analogiques (clarinette classique et logiques) dit en temps réel n'ont pas de temps de latence. La synthèse sonore fonctionne presque en temps-réel mais n'est pas en temps réel. Contrairement au délai geste/son de la clarinette logique, le résonateur virtuel ne fonctionne pas en temps réel, il semble correspondre à la lignée des synthétiseurs. La lignée des synthétiseurs connaît depuis de nombreuses années ce problème que l'on définit par le terme « latence ». II est à noter que cette latence mobilise toutes les énergies créatrices des développeurs dans le domaine de la synthèse numérique pour qu'elle tende vers zéro.

(c) Corine Lévy Soussan

\section{Le régime technologique}

Le mode de la pensée opératoire que développe Anne-Françoise Garçon ${ }^{29}$, s'organise en trois grands régimes : le régime de la pratique qui s'inscrit dans l'oral, le régime de la technique et le régime de la technologie qui s'inscrivent dans l'écrit. Le régime de la pratique « c'est le savoir-faire à l'état brut, tel qu'il est mis en œuvre et transmis par les artisans. Le second régime qui est celui de la technique est issue de l'analyse des pratiques. Le troisième régime, celui de la technologie exprime la nécessité d'un discours rationnel sur la technique et les modes de production ${ }^{30}$. Le régime technologique ainsi exprimé, caractérisé par l'innovation, conjugue en même temps oralité et pratique. La chaine opératoire de la clarinette logique/synthèse sonore s'inscrit dans le régime de pensée technologique. Elle associe connaissance théorique et savoirs pratiques pour concevoir la totalité du système : la composition, la fabrication et l'utilisation d'un produit. Les chercheurs ont inventé des dispositifs qui font appel à un corpus théorique commun basé sur la physique et l'informatique.

\section{Proposition d'une fiche d'inventaire pour la clarinette logique, instrument en développement}

S'agissant d'un instrument en développement il paraît alors nécessaire de compléter la fiche d'inventaire classique en faisant apparaître selon les cas pour chaque instrument, l'historique de sa création, les formules expliquées, un descriptif du logiciel utilisé avec 
les codes sources, les publications, les brevets, les rapports d'étapes et le produit fabriqué dans la chaine de production. Cette fiche d'inventaire prend ainsi en compte les objets, les entretiens et le contexte du laboratoire. La proposition de fiche de l'étude du bec de la clarinette logique en lien avec la synthèse sonore est alors la suivante :

A - Présentation photographique des becs, du résonateur de la clarinette et de l'ensemble complet du projet de la synthèse en lien avec la clarinette logique comme objet de recherche.

B - Information sur la constitution de l'équipe Son du LMA, intérêt de projet clarinette logique et documentation.

C - Principe scientifique et description technique du bec instrumenté contenant des capteurs de pression, de vitesse, les calculs du bec (fabricant, date de fabrication, mesures, matériaux, etc.).

D - Historique du projet : la première collaboration entre le facteur d'instruments à vent, Buffet Crampon et le CNRS remonte à 1987.

$\mathrm{E}$ - La modélisation numérique : le prototype de la clarinette logique est conçu à partir de calculs modélisant les composantes de l'instrument. Calculs effectués à partir de trois équations du modèle global : elles permettent de construire et de modéliser en détail le fonctionnement des becs de clarinette.

F - L'instrument virtuel, la synthèse sonore est la production du son grâce à l'ordinateur. La « synthèse sonore » occupe depuis les années 1960 une place importante parmi les outils dont dispose le musicien : le logiciel utilisé.

G - Publications scientifiques : La base de données PATSTEC côté public ne présente pas le champ documentation qui fait référence aux articles parus dans les diverses publications

H - L'apport des instruments scientifiques contemporains pour l'histoire des techniques, la lignée technique des instruments, les ruptures technologiques.

La méthode proposée inclut le répertoire des éléments logiciels et informatiques : à ce titre, elle s'inscrit dans le cadre de la conservation du patrimoine numérique. Nous nous interrogeons enfin sur la dématérialisation du patrimoine et sur la pérennité des données. En voulant inventorier, référencer etc., on ne peut échapper à la question de la durée du stockage et de la conservation des données. Les données de manière générales seront-elles pérennes? Comment doit-on les conserver? On peut se demander si les données sur disque serveurs etc. ne sont pas vouées à disparaitre? Doit-on commencer à réfléchir à un autre mode de stockage?

\section{Conclusion}

L'étude du dispositif clarinette logique / synthèse sonore met en évidence deux voies de recherche. La première situe les instruments scientifiques dans leur environnement, c'est-à-dire dans le cadre du laboratoire de recherche et des chercheurs, la seconde consiste à insérer les instruments dans la chronologie de l'histoire des techniques au moyen des lignées techniques, ici la lignée des résonateurs acoustiques. A ce niveau il apparait que l'aspect synthèse sonore peut être à l'origine d'une nouvelle lignée technologique basée sur les progrès du calcul numérique qui pourrait contribuer à la diminution du temps de latence.

Le protocole du PATSTEC classique peut être complétée afin de prendre en compte les instruments en développement, par l'ajout des rubriques énoncés ci-dessus y compris 
des informations spécifiques à l'informatique comme les logiciels, les codes sources ou encore les évolutions des algorithmes. De fait, une méthode adaptée à ce type d'instruments devrait inclure tous ces éléments qui ne sont pas classiquement répertoriés. Nous avons proposé un modèle de fiche pour l'inventaire d'un instrument en développement, en tentant d'être le plus exhaustif possible pour la compréhension de sa conception, de son histoire et de son évolution. Cependant, étant donnée la diversité des cas à référencer, il faudrait tester cette approche sur plusieurs cas afin de valider notre type de fiche et éventuellement l'améliorer, le compléter ou le modifier. Une autre difficulté se présente qui est celle de passer d'une pratique courante à une analyse critique et à une réflexion sur la méthodologie. En effet, l'étude de cas a fait émerger des questions de méthodologies (le problème des composants de l'instrument), mais aussi des questions de fond, relatives à la conservation du patrimoine que représentent les instruments scientifiques en évolution. Il est tentant de faire un parallèle avec les objets absents physiquement comme ceux du patrimoine astronomique dont on a seulement des données numériques. L'inventaire d'une soixantaine de missions d'exploration spatiale passées ou qui vont avoir lieu dans un avenir proche qui a été réalisé cette année, montre qu'il existe rarement d'instruments patrimonialisés. Ceux-ci restent soit à bord du satellite, soit en orbite terrestre, ou même se consument lors de la rentrée du satellite dans l'atmosphère. Quand les objets techniques ne sont plus visibles comment retracer une mission?

La description et le mode de fonctionnement de l'objet technique qui conjugue matériaux et mesures nous rapprochent de l'invention et des gestes techniques pour en mémoriser l'usage et les reproduire. La fiche numérisée concrétise la connaissance matérielle des objets techniques surtout si elle est capable de restituer les mouvements mécaniques des objets entre eux. Jusqu'à maintenant l'inventaire des objets fonctionnant avec l'informatique ne s'était pas posé. Nous savons que les objets visibles sur la base de données sont voués à disparaître ou à subir des transformations pour la partie informatique (capteurs et logiciels) qui n'arrêtent pas d'évoluer et la matérialité n'est donc qu'une partie secondaire. La clarinette logique fabriquée en 3D est conçue par modules informatiques qui calculent les paramètres physiques qui vont permettre de reconstruire d'autres clarinettes. Il en est de même de la fabrication et de la conception des becs et des bouches artificielles équipés de capteurs du commerce. L'objet physique ne peut pas être reproduit lorsqu'un composant n'est plus disponible (capteur dépassé en performance, méthode de fabrication). Ils évoluent sous des contraintes extérieures. Certains de ces objets sont jetés ou démantelés de façon à réutiliser les composants valides pour d'autres expérimentations. Les matériaux des objets (bois, plastique ou en imprimante 3D) peuvent influencer le jeu du musicien, le geste qui l'accompagne et la couleur du son. Nous proposons quelques pistes qui demandent à être explorées d'un point de vue de l'histoire des techniques et d'une certaine manière de celui de la culture matérielle. Ce travail met en exergue l'intérêt qu'il y aurait à entreprendre un travail continu avec les chercheurs, afin d'être au plus près de leurs travaux.

40 La motivation des chercheurs dans ce travail pourrait être facilité par une démarche de «management de la qualité31 » au sein des laboratoires. De plus en plus de laboratoires utilisent cet outil, à l'aide de normes (norme ISO $9001^{32}$ par exemple) afin d'avoir une traçabilité complète de l'ensemble des processus mis en jeu évitant la perte d'informations. Par exemple pour informer le lieu, les conditions de stockage des échantillons, les protocoles utilisés dans l'expérience, les données etc. Ceci pourrait 
être utilisé afin de faciliter l'inventaire dans un laboratoire. Mais quoi qu'il en soit, la mise en œuvre de cette méthode a pour condition l'implication des chercheurs, qui y ont un rôle central. Il faut alors se demander comment inciter les chercheurs à s'investir dans des activités permettant la conservation et l'inventaire de l'instrumentation scientifique.

41 La proposition ici présentée renvoie à la synthèse de la journée d'étude "Quels nouveaux défis du patrimoine scientifique, technique et industriel ? » dans le cadre de l'année Européenne du patrimoine culturel ${ }^{33}$ qui met en exergue un " point capital » à savoir la question de la mémoire du travail du chercheur et des traces de son action comme condition d'une sauvegarde du patrimoine scientifique de la recherche au XXI siècle. Tel est bien, en effet, le défi, pour lequel notre étude du cas de la clarinette logique ouvre des pistes de recherche pour la patrimonialisation des instruments scientifiques contemporains et en développement.

\section{BIBLIOGRAPHIE}

BRENNI Paolo, SARTONIANA, Volume 14, Sarton Chair of the History of Science, University of Glen, Belgium, Site de l'Université : https://lib.ugent.be/fulltxt/RUG01/000/170/842/BIBG-024341-14_2010_0001_AC.pdf, p.36, 2001, consulté le 22 octobre 2018.

CHAIGNE Antoine, KERGOMARD Jean, Acoustique des instruments de musique, Paris, Édition BELIN, 2013.

CUENCA Catherine, BALLÉ Catherine, THOMAS Yves, Patrimoine Scientifique et technique : un projet contemporain, Paris, La documentation Française, 2010.

CUENCA Catherine, «Le patrimoine scientifique et technique du $\mathrm{XX}^{\mathrm{e}}$ siècle : un projet de sensibilisation et de sauvegarde », Culture \& Musées, n², 203, p.129-143. http://www.persee.fr/ doc/pumus_1766-2923_2003_num_2_1_1181, consulté le 18 janvier 2019.

DUBOURG-GLATIGNY Pascal et VÉRIN Hélène (dir.), Réduire en Art. La technologie de la Renaissance aux Lumières, Paris, éd. MSH, 2008.

ESCHALIER Nicolas, Comprendre la latence, site en ligne « Devenir INGESON » : https:// deveniringeson.com/comprendre-la-latence/ , 2017, consulté le 20 juillet 2018, accès libre et gratuit.

GARÇON Anne-Françoise, site de l'auteure : https://sites.google.com/site/afgarcon/home, consulté le 22 juin 2018.

GARÇON Anne-Françoise, L'Imaginaire et la pensée technique. Une approche historique, $\mathrm{XVI}^{\mathrm{e}}-\mathrm{XX}^{\mathrm{e}}$ siècle, Paris, Classiques Garnier, 2012.

JACOMY Bruno, Une histoire des techniques, Paris, Éd. du Seuil, 1990.

LEBEAUME Joël, L'introduction de l'évolution de l'objet technique au collège : analyse didactique, revue en ligne : recherche en didactique des sciences et des technologies (RDST), 2011 : https:// journals.openedition.org/rdst/401, consulté en juin 2018. 
LÉVY-SOUSSAN Corine, Le patrimoine instrumental de la Recherche (Seconde moitié du XX $\mathrm{X}^{\mathrm{e}}$ et début du XXI ${ }^{\mathrm{e}}$ siècle) : Inventaire et sauvegarde des instruments scientifiques en développement, mémoire de Master 2 Recherche : Techniques, Patrimoine, Territoires de l'Industrie, soutenance à Paris 1 Panthéon Sorbonne, 2018.

RAINETTE Caroline, en collaboration et sous la direction scientifique de CORNU Marie et WALLAERT Catherine, Guide juridique sur le patrimoine scientifique et technique, Paris, L'Harmattan, 2008, p.67.

RISSET Jean-Claude, Le son numérique : une acoustique affranchie de la mécanique ? 1992, [En ligne] https://hal.archives-ouvertes.fr/jpa-00251043/document, consulté le 23 février 2018.

SIMONDON Gilbert, Genèse de l'objet technique : le processus de concrétisation, Du mode d'existence des objets techniques, Paris, Éd. Aubier, 1958.

VERGEZ Christophe, Trompettes et trompettiste, un système dynamique, non linéaire ... et musical, présentation du travail de thèse, 2000.

WIKIN Yves, Le programme de sauvegarde et de valorisation du patrimoine scientifique et technique contemporain, lettre de l'OCIM, en ligne, https://journals.openedition.org/ocim/1691\#tocto1n10, octobre 2016, consulté le 20 juin 2018.

\section{NOTES}

1. Cet article est basé sur une recherche menée au sein de l'équipe d'Histoire des techniques de l'Université de Paris 1, dans le cadre du Master 2 Recherche (TPTI). Il synthétise les résultats présentés dans mon mémoire, Le patrimoine instrumental de la Recherche (Seconde moitié $d u X^{e}$ et début du XXI siècle) - Inventaire et sauvegarde des instruments scientifiques en développement, réalisé sous la direction de Anne-Françoise GARÇON.

2. «Recherche et Développement» (R\&D), https ://fr.wikipedia.org/wiki/ Recherche_et_d\%C3\%A9veloppement, consulté en ligne le 20 juin 2018.

3. «Aujourd'hui la justesse est un souci majeur (notons que la définition de la justesse optimale n'est pas du tout évidente du point de vue musical), mais aussi l'homogénéité, d'émission comme de timbre. Partant de critères comme ceux-ci, nous proposons de définir l'ensemble des trous latéraux et éventuellement les changements de section du tube satisfaisant ces critères. Les connaissances en acoustique musicale ont déjà permis de résoudre des problèmes ponctuels de facture instrumentale, il s'agit ici d'envisager une conception globale. Nous appellerons instruments " logiques " des instruments justes et homogènes conçus de façon globale ". Entretien avec Philippe GUILLERMAIN, le 18 février 2018.

4. CUENCA Catherine, Le patrimoine scientifique et technique du $\mathrm{XX}^{\mathrm{e}}$ siècle: un projet de sensibilisation et de sauvegarde dans Culture \& Musées, $\mathrm{n}^{\circ} 2,203$, Musées et organisation (sous la direction de Catherine Ballé), P129-143. $\underline{\text { http://www.persee.fr/doc/ }}$ pumus_1766-2923_2003_num_2_1_1181, consulté en ligne le 18 janvier 2019.

5. «Les objets évoluent selon les « lignées ", c'est-à-dire des ensembles regroupent les objets de même type, ayant même fonction et même principe de fonctionnement », JACOMY Bruno, Une histoire des techniques, édit. Du Seuil, Paris,1990.

6. GARÇON Anne-Françoise, L'imaginaire et la pensée technique. Une approche historique, XVI ${ }^{e}-X^{e}$ siècle, Paris, Classique Garnier, 2012.

7. BRENNI Paolo, SARTONIANA, Sarton Chair of the History of Science, University of Glen, Belgium, p. 36, 2001, https://lib.ugent.be/fulltxt/RUG01/000/170/842/BIB-G-024341-14_2010_0001_AC.pdf, consulté en ligne le 22 octobre 2018. 
8. WIKIN Yves, Le programme de sauvegarde et de valorisation du patrimoine scientifique et technique contemporain, 2016, en ligne : https://journals.openedition.org/ocim/1691\#tocto1n10, consulté le 23 juillet 2018.

9. Philippe GUILLEMAIN, ibidem.

10. VERGEZ Christophe, Trompettes et trompettiste, un système dynamique, non linéaire ... et musical, présentation du travail de thèse, 2000.

11. Philippe GUILLEMAIN est coordinateur des deux projets et directeur de recherche au CNRS.

12. "L'embouchure constituée du bec et de l'anche : l'anche agit comme une valve qui module le débit d'air produit entre l'amont (la bouche de l'instrumentiste) et l'aval (l'embouchure ou le bec de l'instrument - C'est le générateur de son) », Philippe GUILLERMAIN, Ibid.

13. Synthèse d'entretien avec Christophe VERGEZ Christophe, le 15 octobre 2017. Directeur de recherche au CNRS, il s'intéresse aux bouches artificielles.

14. Synthèse d'entretien avec Philippe GUILLEMAIN, le 15 octobre 2017.

15. La perce est la forme intérieure d'un tuyau d'un instrument à vent. C'est dans la perce que vibre la colonne d'air qui détermine les caractéristiques et la hauteur d'un son musical. La clarinette (source) : https://fr.wikipedia.org/wiki/Perce, consulté en ligne le 18 janvier 2018.

16. Entretien avec Philippe GUILLEMAIN, Juin 2018.

17. Le perçage est un usinage consistant à faire un trou dans une pièce, Ibidem.

18. Voir figure 5.

19. GUILLEMAIN Philippe, ibidem.

20. D'après CHAIGNE Antoine, KERGOMARD Jean, Acoustique des instruments de musique, Paris, Édition Belin, Paris, 2013. Source des travaux : OLLIVIER Sébastien, Contribution à l'étude des oscillations des instruments à vent à anche simple, Thèse Doctorat en Acoustique, sous la direction de KERGOMARD Jean, Université du Maine (Le Mans), 2004.

21. Synthèse d'entretiens avec Philippe GUILLEMAIN, octobre 2017.

22. Le logiciel Max/MSP a été développé par l'Ircam en 1980, https://fr.wikipedia.org/wiki/Max/ MSP, consulté en ligne le 20 juin 2018.

23. LEBEAUME Joël, L'introduction de l'évolution de l'objet technique au collège: analyse didactique, 2011, revue : recherche en didactique des sciences et des technologies (RDST), en ligne : https:// journals.openedition.org/rdst/401, consulté le 14 juillet 2018.

24. Ibidem.

25. SIMONDON Gilbert, Genèse de l'objet technique: le processus de concrétisation, Du mode d'existence des objets techniques, Paris, Éd. Aubier, 1958.

26. Synthèse d'entretiens avec Claude Gazanhes, directeur du LMA de 1986 à 1992, juillet 2018.

27. Voir figure 6.

28. Le temps de latence dans le domaine de la synthèse du son c'est le délai qui s'écoule entre le geste du musicien et la production du son physique dans le haut-parleur. Voir à ce sujet ESCHALIER Nicolas, Comprendre la latence, 2017, en ligne: https://deveniringeson.com/ comprendre-la-latence/, consulté le 20 juillet 2018.

29. GARÇON Anne-Françoise, L'Imaginaire..., op.cit., 2012, p.26.

30. Cours en ligne sur le blog d'Anne-Françoise GARÇON, https://sites.google.com/site/afgarcon/ home, consulté le 22 juin 2018.

31. Système de management de la qualité : https://fr.wikipedia.org/wiki/ Syst\%C3\%A8me_de_management_de_la_qualit\%C3\%A9, consulté le 24 juillet 2018.

32. Norme ISO 9001 : Organisation internationale de normalisation.

33. Journée d'étude sur le patrimoine scientifique, technique et industriel du 15 novembre 2018, organisée à Paris par le ministère d la Culture (département de la recherche, de l'enseignement supérieur et de la technologie) et l'office de coopération et d'information muséales (OCIM). 


\section{RÉSUMÉS}

La question étudiée est celle de l'inventaire et de la conservation des instruments scientifiques qui sont l'objet même d'une recherche, donc intrinsèquement évolutifs, autrement dit 'en développement'. Le contexte est celui de la mission nationale PATSTEC (Patrimoine Scientifique et Technique Contemporain) et son inscription dans l'Université d'Aix-Marseille (AMU). La méthodologie d'inventaire des instruments scientifiques classiques telle que mise en œuvre dans le cadre de la mission PATSTEC montre ses limites dès lors qu'on veut traiter d'instruments 'en développement'. Est alors proposé un protocole spécifique, qui passe par une mise en histoire de la recherche dont l'instrument scientifique est l'émanation. L'étude de cas permet de mettre en œuvre le protocole pour le cas de la 'clarinette logique', projet de recherche du Laboratoire de Mécanique et d'Acoustique (LMA) de l'AMU. L'étude de cas fait émerger des questions méthodologiques (le problème des composantes de l'instrument), mais aussi des questions de fond, relatives à la conservation du patrimoine que représentent les instruments scientifiques dans la perspective élargie proposée ici (par exemple le statut des logiciels). Ces éléments constituent autant de pistes pour des recherches futures.

The research addresses the issue of the inventory and conservation of scientific instruments which are themselves an object of research, instruments which we call 'in development'. The context of the study is the PATSTEC (contemporary scientific and technical heritage) national mission and its activity in the Aix-Marseilles University (AMU). The inventory methodology as implemented in the PATSTEC mission appears ill-suited for such instruments in development. A specific protocol is proposed which consists in placing in historical context the research within which the scientific instrument is being developed. The protocol is implemented in a case study concerning the 'logical clarinette', a research project of the Mechanics and Acoustics Laboratory (LMA) of the AMU. The case study reveals further methodological issues (for example the treatment of the components of the instruments), but also more general issues related to the conservation of the instruments in the broader perspective proposed here (for example the softwares). These issues are perspectives for future research.

\section{INDEX}

Mots-clés : histoire des techniques, histoire des sciences, patrimoine scientifique, culture matérielle, patrimoine immatériel, XXe siècle, XXIe siècle

Index chronologique : Époque contemporaine

Index géographique : France

Keywords : history of technology, history of science, scientific heritage, intangible heritage, XXth century, XXIst century

\section{AUTEUR}

\section{CORINE LÉVY-SOUSSAN}

Chargée de l'inventaire du patrimoine scientifique et technique contemporain (PATSTEC) d'AixMarseille Université (AMU), Corine Lévy-Soussan est titulaire d'un Magister Sciences et Techniques dans la Société du Conservatoire National des Arts et Métiers (CNAM) et d'un Master Recherche Techniques, Patrimoines, Territoires de l'Industrie (TPTI) de Paris 1 Panthéon 
Sorbonne. Ses travaux de recherche se sont développés dans le cadre du département d'Histoire des Techniques de Paris I Panthéon Sorbonne sous la direction d'Anne-Françoise Garçon ; ils portent sur l'inventaire et la conservation des instruments scientifiques en développement, qui doivent être appréhendés dans leur dynamique d'évolution, en termes de lignée technique. Cet article est issu du mémoire intitulé : Le patrimoine instrumental de la Recherche (Seconde moitié du XXe et début du XXIe siècle) : Inventaire et sauvegarde des instruments scientifiques en développement. Corine Lévy-Soussan valorise et diffuse ses travaux d'inventaire réalisés au sein du programme national et régional de sauvegarde du patrimoine scientifique et technique contemporain (PATSTEC) sous forme de films, vitrines d'exposition, catalogues et livrets sur l'évolution des instruments scientifiques pour des publics variés. Elle participe à l'exposition, prévue en 2020, sur les « Prototypes » (objets techniques de la recherche) et au catalogue de l'exposition, portée par la mission nationale qui comprend des partenaires régionaux et nationaux (universités, entreprises, délégations des CNAM en région, structures culturels, scientifiques et techniques dont les muséums ...). 